\title{
Occult Hip Fractures in Elderly Patients: Is CT Enough?
}

\section{Andy KH Lim ${ }^{1,2 *}$}

${ }^{1}$ Department of Nephrology, Monash University, Monash Medical Centre, 246 Clayton Road, Clayton VIC 3168, Australia

${ }^{2}$ Gen Med (Los Angel) icine, Dandenong Hospital, 105 David Street, Dandenong VIC 3175, Australia

Keywords: Hip fracture; Occult fracture; Elderly; Imaging; Magnetic resonance imaging; Computed tomography

An elderly patient who presents with a painful hip following a fall or low-level trauma is an increasingly common scenario faced by Gen Med (Los Angel) icine physicians. In the past, almost all such patients were evaluated and diagnosed in the Emergency Department. In the modern era of rapid transit through our Emergency Departments and the advent of acute medical assessment units, many general physicians will be faced with such patients. General physicians will require the necessary skills to confidently exclude occult hip fractures and understand the pros and cons of various imaging modalities.

It is well-known that undisplaced hip fractures may not manifest any radiographic abnormality on plain X-rays in elderly patients. The prevalence of occult hip fractures is estimated at 3-10\% [1,2]. Additional risk factors for missed hip fractures include age $>70$ years, poor baseline mobility and cognitive impairment $[3,4]$. These occult fractures are often diagnosed with second line imaging such as a bone scan, CT or MRI scan. The potential consequences of missing occult fractures include a delay in definitive treatment, displacement of previously undisplaced fracture and prolonged non-weight bearing status leading to physical deconditioning [5]. Early and accurate diagnosis is also important as a delay in operative treatment is associated with increased mortality [6].

In an ideal word, universal MRIs could be utilised for these patients given its excellent track record with near $100 \%$ accuracy and good inter-observer agreement [7]. The main limitation of a bone scan is the early high false negative rate if performed within the first 72 hours of injury. Bone scanning also lacks the resolution for accurate anatomic localisation. CT scans have been shown to be superior to plain X-rays in diagnosing hip fractures [8]. They are cost-effective, readily available, are quick to perform, and have fewer contraindications. In many centres, CT is the preferred second line investigation for these reasons. However, there is a paucity of data directly comparing the performance of CT with MRI for occult hip fractures in the elderly.

Lubovsky et al. compared CT and MRI in the diagnosis of traumaassociated hip pain in elderly patients [9]. The MRI study involved T1weighted spin echo and Short Tau Inversion Recovery (STIR) axial and coronal scans. The authors evaluated 13 patients with suspected hip fracture after a fall. The mean age of these patients was 73 years. Six patients had both CT and MRI (Group A) and 7 patients had MRI alone (Group B). In group A, CT did not miss a fracture but misclassified the fracture in $4 / 6$ patients (e.g., greater trochanteric vs. intertrochanteric). In group $B, 1 / 6$ was diagnosed with a subcapital fracture and the others had acetabular or soft tissue injury. Compared to patients who had sequential CT and MRI scans, the average time to achieve a final diagnosis was 48 hours less if MRI was chosen upfront. The authors suggest that MRI affords better accuracy and changed the treatment in $62 \%$ of patients. This translated into earlier ambulation for patients without a fracture and helped define the type of operation for those with a fracture. MRI also eliminates the need for repeated or supplemental imaging. The limitations include the small, retrospective nature of the study and a lack of inter-observer correlation.

The recent study by Hakkarinen et al. also attempted to evaluate the performance of CT and MRI for occult fractures [1]. The MRI study involved T1- and T2-weighted and proton density images with fat saturation; with axial, coronal and sagittal images. In this larger retrospective study, the authors evaluated the imaging results of 235 patients over the age of 60 years with a final diagnosis of hip fracture. In this cohort, $10 \%$ of fractures were occult and $1.7 \%$ had a negative CT but positive MRI. CT missed two femoral neck fractures, one intertrochanteric fracture and one acetabular fracture. Three of these patients were treated operatively. Thus, MRI was able to detect $20 \%$ of occult hip fractures missed on CT and changed the management for most of these patients.

With these retrospective studies, the true number of CT misses is hard to determine without simultaneous MRI scanning in all patients. The number of occult fractures may be underappreciated as many patients with normal X-rays or CT do not acquire further imaging. The limited data so far suggests that CT is not definitive in excluding occult hip fracture in elderly patients. However, it does pick up the majority. It is hypothesised that the generalised radiolucency associated with osteoporotic bones in the elderly lowers the sensitivity of CT. This is the basis for the algorithm suggested by Cannon et al. where patients with osteoporosis risk factors and low-energy trauma are stratified to receive MRI rather than CT [2].

There may be a cost benefit with early upfront MRI given the potential for early ambulation and shorter hospitalisation [7]. However, health care services should perform their own cost-benefit analysis using local data and expertise. Furthermore, a limited MRI sequence may be sufficient for diagnosing hip fractures and allows a shorter scanning time. Using T1-weighted coronal scans with STIR sequences, Iwata et al. reported sensitivities approaching 100\% [10]. Others have suggested that T1-weighted images alone are sufficient, with scanning times down to 15 minutes. However, this approach may miss soft tissue injuries. Further validation of the limited MRI approach is required.

With further improvements in CT scanning protocols and 128-slice CT scanners, larger prospective studies would be useful to obtain robust comparisons. For now, CT is a reasonable next step for detecting occult fractures in the elderly but more data is certainly needed before one can argue that "CT is enough". MRI remains the clinical gold standard for excluding occult hip fractures in elderly patients and if a cost-benefit can be demonstrated, the case for an MRI upfront could be entirely justified.

*Corresponding author: $\mathrm{Dr}$ Andy $\mathrm{KH}$ Lim, MBBS, FRACP, PhD Department of Nephrology, Monash Medical Centre, 246 Clayton Road, Clayton VIC 3168 Australia, Tel: +61 39594 6666; Fax: + 6139594 6530; E-mail: andy.lim@monash.edu

Received February 26, 2013; Accepted February 28, 2013; Published March 04, 2013

Citation: Andy KH (2013) Occult Hip Fractures in Elderly Patients: Is CT Enough? Gen Med (Los Angel) 1: e105. doi: 10.4172/2327-5146.1000e105

Copyright: (๑) 2013 Andy $\mathrm{KH}$. This is an open-access article distributed under the terms of the Creative Commons Attribution License, which permits unrestricted use, distribution, and reproduction in any medium, provided the original author and source are credited. 